\title{
PARABOLIC EQUATIONS IN A SINGULARLY TIME DEPENDENT DOMAIN
}

\author{
Jamil V. Pereira*, Ricardo P. Silva ${ }^{\dagger}$ \\ *UNESP - Universidade Estadual Paulista "Júlio de Mesquita Filho", Instituto de Geociências e \\ Ciências Exatas, \\ Departamento de Matemática, Rio Claro SP, Brazil \\ †UNESP - Universidade Estadual Paulista "Júlio de Mesquita Filho", Instituto de Geociências e \\ Ciências Exatas, \\ Departamento de Matemática, Rio Claro SP, Brazil
}

Emails: jamil@rc.unesp.br, rpsilva@rc.unesp.br

\begin{abstract}
We study the limiting regime of nonlinear parabolic equations posed in a time dependent family of domains $\left\{\Omega_{t}^{\epsilon}\right\}_{t \in \mathbb{R}} \subset \mathbb{R}^{n+1}$ which collapses to a lower dimensional set as the parameter $\epsilon$ goes to 0 .
\end{abstract}

Keywords - evolution process, thin domains, noncylindrical domain

\section{Introduction}

In this work, based on paper [5], we are concerned with the effective behavior of a nonlinear reactiondiffusion equation posed in a time dependent family of domains which collapses to a lower dimensional set. Inspired by the recent works [3,4], both of them related with asymptotic behavior of reaction-diffusion equations on time-dependent domains, we are interested with reaction-diffusion equations in a time dependent thin domain. For the best of our knowledge this is an untouched topic in the literature.

In order to set up the problem, let $\omega$ be a smooth bounded domain in $\mathbb{R}^{n}, n \geq 1$, and $g \in$ $C^{2}(\bar{\omega} \times \mathbb{R} ; \mathbb{R})$ satisfying

$\left(G_{1}\right)$ There exist positive constants $\alpha_{1}$ and $\alpha_{2}$ such that

$$
\alpha_{1} \leq g,\left|\nabla_{x} g\right|, g_{t}, g_{t t} \leq \alpha_{2}
$$

for all $(x, t) \in \omega \times \mathbb{R}$;

$\left(G_{2}\right)$ There exists a constant $k$ such that

$$
\left|g_{x_{i}}(x, t)-g_{x_{i}}(x, s)\right| \leq k|t-s|,
$$

for all $(x, t) \in \omega \times \mathbb{R}, i=1,2, \cdots, n$.

In the following, $\epsilon$ will denote a positive parameter which will converge to zero. Fixed $t \in \mathbb{R}$ we define the time-dependent thin domain

$$
\Omega_{t}^{\epsilon}:=\left\{(x, y) \in \mathbb{R}^{n+1}: x \in \omega, 0<y<\epsilon g(x, t)\right\} .
$$

For each $\tau \in \mathbb{R}$ and $\epsilon \geq 0$ we set the domain

$$
Q_{\tau}^{\epsilon}:=\bigcup_{t \in(\tau, \infty)} \Omega_{t}^{\epsilon} \times\{t\}
$$

as well the lateral boundary

$$
\Sigma_{\tau}^{\epsilon}:=\bigcup_{t \in(\tau, \infty)} \partial \Omega_{t}^{\epsilon} \times\{t\},
$$

where $\Omega_{t}^{0}:=\omega$ for all $t \in(\tau, \infty)$.

For positive values of the parameter $\epsilon$, we consider the semilinear reaction-diffusion equation

$$
\left\{\begin{array}{l}
u_{t}^{\epsilon}-\Delta u^{\epsilon}+u^{\epsilon}=f\left(u^{\epsilon}\right), \quad \text { in } Q_{\tau}^{\epsilon}, \\
\frac{\partial u^{\epsilon}}{\partial \eta_{\tau}^{\epsilon}}=0, \quad \text { on } \Sigma_{\tau}^{\epsilon}, \\
u^{\epsilon}(\cdot, \tau)=u_{\tau}^{\epsilon}, \quad \text { in } \Omega_{\tau}^{\epsilon},
\end{array}\right.
$$

where $\eta_{\tau}^{\epsilon}$ denotes the unit outward normal vector field to $\Sigma_{\tau}^{\epsilon}, \frac{\partial}{\partial \eta_{\tau}^{\epsilon}}$ denotes the outwards normal derivative and $f: \mathbb{R} \rightarrow \mathbb{R}$ is a $C^{2}$-function with bounded derivatives up second order.

Besides, since our interest resides in the asymptotic behavior of the solutions and its dependence with respect to $\epsilon$, we will require that solutions of (1) are bounded for large values of time. A natural assumption to obtain this boundedness is expressed in the following dissipative condition

$$
\limsup _{|s| \rightarrow \infty} \frac{f(s)}{s}<0
$$

This implies for any $\eta>0$, the existence of a positive constant $c_{\eta}$ such that,

$$
f(s) s \leq \eta s^{2}+c_{\eta}, \quad \forall s \in \mathbb{R} .
$$

In the analysis of the limiting behavior of the problem (1), it will be useful to introduce the domain $\Omega:=\omega \times(0,1)$, independent of $\epsilon$ and $t$, which is obtained from $\Omega_{t}^{\epsilon}$ by the change of coordinates

$$
\begin{aligned}
\mathcal{T}_{t}^{\epsilon}: \Omega & \rightarrow \Omega_{t}^{\epsilon} \\
(x, y) & \mapsto(x, \epsilon g(x, t) y)
\end{aligned}
$$

Such change of coordinates induces an isomorphism from $W^{m, p}\left(\Omega_{t}^{\epsilon}\right)$ onto $W^{m, p}(\Omega)$ by

$$
u \stackrel{\Phi_{t}^{\epsilon}}{\longmapsto} v:=u \circ \mathcal{T}_{t}^{\epsilon}
$$


with partial derivatives related by

$$
\begin{aligned}
& u_{t}=v_{t}-\frac{y g_{t}}{g} v_{y} \\
& u_{x_{i}}=v_{x_{i}}-\frac{y g_{x_{i}}}{g} v_{y}, \quad i=1, \ldots, n \\
& u_{y}=\frac{1}{\epsilon g} v_{y} .
\end{aligned}
$$

In this new coordinates we rewrite equation (1) as the following (nonautonomous) equation posed in the fixed domain $\Omega$,

$$
\left\{\begin{array}{l}
v_{t}^{\epsilon}-\frac{1}{g} \operatorname{div} B_{\epsilon}(t) v^{\epsilon}-y \frac{g_{t}}{g} v_{y}^{\epsilon}+v^{\epsilon}=f\left(v^{\epsilon}\right), \quad \text { in } \Omega, \\
B_{\epsilon}(t) v^{\epsilon} \cdot \eta=0, \quad \text { on } \partial \Omega, \\
v^{\epsilon}(\cdot, \tau)=u_{\tau}^{\epsilon} \circ \mathcal{T}_{\tau}^{\epsilon}, \quad \text { in } \Omega,
\end{array}\right.
$$

where $\eta$ denotes the unit outward normal vector field to $\partial \Omega$, and

$$
B_{\epsilon}(t) v=\left[\begin{array}{c}
g v_{x_{1}}-y g_{x_{1}} v_{y} \\
\vdots \\
g v_{x_{n}}-y g_{x_{n}} v_{y} \\
-\sum_{i=1}^{n} y g_{x_{i}} v_{x_{i}}+\frac{1}{\epsilon^{2} g}\left(1+\sum_{i=1}^{n}\left(\epsilon y g_{x_{i}}\right)^{2}\right) v_{y}
\end{array}\right]
$$

After a careful study of the solutions of (5), one starts to suspect that $v^{\epsilon}$ tends not to depend on the variable $y$ as $\epsilon \rightarrow 0$. Therefore, if a limiting regime for the problem (1) exists, then it should be given by the (non-autonomous) problem

$$
\left\{\begin{array}{l}
v_{t}-\frac{1}{g} \sum_{i=1}^{n}\left(g v_{x_{i}}\right)_{x_{i}}+v=f(v), \quad \text { in } \omega \\
\frac{\partial v}{\partial \nu}=0, \quad \text { on } \partial \omega \\
v(\cdot, \tau)=v_{\tau}, \quad \text { in } \omega
\end{array}\right.
$$

where $\nu$ denotes the unit outward normal vector field to $\partial \omega$.

The comparison between solution of the problems (5) and (6) is the aim of this paper.

\section{Abstract Formulation}

We start stressing the fact that $\Omega_{t}^{\epsilon}$ varies in accordance with a positive parameter $\epsilon$, collapsing themselves to the lower dimensional domain $\omega$ as $\epsilon$ goes to 0 . Therefore, in order to preserve the "relative capacity" of a mensurable subset $E \subset \Omega_{t}^{\epsilon}$, we rescale the Lebesgue measure by a factor $1 / \epsilon$ dealing with the singular measure $\rho^{\epsilon}(E)=\epsilon^{-1}|E|$.

As we will see, it will be convenient to consider the space $H_{\epsilon}:=H^{1}(\Omega)$ endowed with the equivalent norm

$$
\|v\|_{H_{\epsilon}}:=\left[\int_{\Omega}\left(\left|\nabla_{x} v\right|^{2}+\frac{1}{\epsilon^{2}}\left|v_{y}\right|^{2}+|v|^{2}\right) d x d y\right]^{\frac{1}{2}} .
$$

It is immediate consequence of $\left(G_{1}\right)$ that the family of isomorphism $\left\{\Phi_{t}^{\epsilon}\right\}$ satisfies

$$
\left\|\Phi_{t}^{\epsilon}\right\|_{\mathcal{L}\left(H^{1}\left(\Omega_{t}^{\epsilon} ; \rho^{\epsilon}\right), H_{\epsilon}\right)} \leq c,
$$

for some positive constant $c$ independent on $\epsilon$ and $t$.

For each pair of parameters $(\epsilon, t) \in(0,1] \times \mathbb{R}$, we consider the sesquilinear form

$$
\begin{gathered}
a_{t}^{\epsilon}: H_{\epsilon} \times H_{\epsilon} \rightarrow \mathbb{R} \\
a_{t}^{\epsilon}(u, v)=\int_{\substack{\Omega \\
+}}\left(B_{\epsilon}(t) u \cdot \nabla v-y g_{t}(x, t) u_{y} v\right. \\
+g v) d x d y
\end{gathered}
$$

As a first remark, we notice that under assumptions $\left(G_{1}\right), a_{t}^{\epsilon}$ is a continuous form and there exist positive constants $c_{1}, c_{2}$, independents on $\epsilon$ and $t$, such that

$$
c_{1}\|v\|_{H_{\epsilon}}^{2} \leq a_{t}^{\epsilon}(v, v) \leq c_{2}\|v\|_{H_{\epsilon}}^{2},
$$

for all $v \in H_{\epsilon}$ and $(\epsilon, t) \in(0, \bar{\epsilon}] \times \mathbb{R}$.

Since $H_{\epsilon}$ is densely and compactly embedding in $L^{2}(\Omega)$, the sesquilinear form $a_{t}^{\epsilon}$ yields a densely defined positive linear operator with compact resolvent, $A_{\epsilon}(t): D\left(A_{\epsilon}(t)\right) \subset L^{2}(\Omega) \rightarrow L^{2}(\Omega)$, which is defined by the relation

$a_{t}^{\epsilon}(u, v)=\left(A_{\epsilon}(t) u, v\right)_{t}, \quad u \in H_{\epsilon}, v \in D\left(A_{\epsilon}(t)\right)$,

where $(u, v)_{t}:=\int_{\Omega} g(x, t) u v d x d y$.

By regularity of $\partial \omega$ we notice that

$$
D\left(A_{\epsilon}(t)\right)=\left\{v \in H^{2}(\Omega): B_{\epsilon}(t) v \cdot \eta=0\right\},
$$

is independent on $\epsilon$. Moreover

$$
A_{\epsilon}(t) v=-\frac{1}{g(\cdot, t)} \operatorname{div} B_{\epsilon}(t) v-y \frac{g_{t}(\cdot, t)}{g(\cdot, t)} v_{y}+v,
$$

for all $v \in D\left(A_{\epsilon}(t)\right)$.

Multiplying equation (5) by $\varphi \in H^{1}(\Omega)$ and integrating by parts we get

$$
\left(v_{t}^{\epsilon}, \varphi\right)_{t}+a_{t}^{\epsilon}\left(v^{\epsilon}, \varphi\right)=\left(f\left(v^{\epsilon}\right), \varphi\right)_{t} .
$$

Hence we can write equation (5) as an abstract equation

$$
\frac{d v^{\epsilon}}{d t}(t)+A_{\epsilon}(t) v^{\epsilon}(t)=f^{e}\left(v^{\epsilon}(t)\right)
$$

where $f^{e}$ is the Nemitskii operator (composition operator) associated to $f$.

Combining assumptions $\left(G_{1}\right)-\left(G_{2}\right)$, we also have that

$$
\left|a_{t}^{\epsilon}(u, v)-a_{s}^{\epsilon}(u, v)\right| \leq k_{1}|t-s|\|u\|_{H_{\epsilon}}\|v\|_{H_{\epsilon}},
$$

for some constant $k_{1}$ independent on $\epsilon, t, s \in \mathbb{R}$, and $u, v \in H_{\epsilon}$. Therefore, thanks to Theorem 5.4 .2 in [6] there exists an unique solution of the linear homogeneous problem

$$
\left\{\begin{array}{l}
\frac{d v^{\epsilon}}{d t}(t)+A_{\epsilon}(t) v^{\epsilon}(t)=0, \quad t \geq \tau \in \mathbb{R} \\
v^{\epsilon}(\tau)=v_{\tau}^{\epsilon} \in H_{\epsilon}
\end{array}\right.
$$


This allow us to consider for each value of the parameter $\epsilon$, each initial time $\tau \in \mathbb{R}$ and each initial data $v_{\tau}^{\epsilon} \in H_{\epsilon}$, the solution $v^{\epsilon}\left(\cdot, \tau, v_{\tau}^{\epsilon}\right) \in$ $C^{1}\left([\tau, \infty) ; H_{\epsilon}\right)$ of $(11)$. This give raise a linear process $\left\{L_{\epsilon}(t, \tau), t \geq \tau\right\} \subset \mathcal{L}\left(H_{\epsilon}\right)$ defined by $L_{\epsilon}(t, \tau) v_{\tau}^{\epsilon}:=v^{\epsilon}\left(t, \tau, v_{\tau}^{\epsilon}\right)$. We notice that (11) is the abstract Cauchy problem associated to the equation (5) in the case $f \equiv 0$.

Since we are assuming the nonlinearity $f \in$ $C^{2}(\mathbb{R} ; \mathbb{R})$ bounded as well as its derivatives up second order, local existence of the nonlinear counterpart is guaranteed by Theorem 6.6.1 in [6], i.e, writing the problem (5) as

$$
\left\{\begin{array}{l}
\frac{d v^{\epsilon}}{d t}(t)+A_{\epsilon}(t) v^{\epsilon}(t)=f^{e}\left(v^{\epsilon}(t)\right), \\
v^{\epsilon}(\tau)=v_{\tau}^{\epsilon} \in H_{\epsilon}
\end{array}\right.
$$

there exist a time $T_{\tau}>0$ and an unique solution $v^{\epsilon}\left(\cdot, \tau, v_{\tau}^{\epsilon}\right) \in C^{1}\left(\left[\tau, \tau+T_{\tau}\right] ; H_{\epsilon}\right)$ of $(12)$. Under dissipative assumption (2) on the nonlinearity $f$, one can show that actually $v^{\epsilon}\left(\cdot, \tau, v_{\tau}^{\epsilon}\right) \in$ $C^{1}\left([\tau, \infty) ; H_{\epsilon}\right)$. Further details can be found in [1] and [2].

Similarly to the linear case, this allow us to consider for each value of the parameter $\epsilon$, each initial time $\tau \in \mathbb{R}$, and each initial data $v^{\epsilon} \in H_{\epsilon}$ , the (nonlinear) evolution process $\left\{S_{\epsilon}(t, \tau): t \geq\right.$ $\tau\}$ in the state space $H_{\epsilon}$ defined by $S_{\epsilon}(t, \tau) v^{\epsilon}:=$ $v^{\epsilon}\left(t, \tau, v^{\epsilon}\right)$.

By reader's convenience we recall the definition of an evolution process in a Banach space

Definition 1 We say that a family of maps $\{S(t, \tau): t \geqslant \tau \in \mathbb{R}\}$ from a Banach space $\mathcal{X}$ into itself is an evolution process if

(i) $S(\tau, \tau)=I$ (identity operator in $\mathcal{X}$ ), for any $\tau \in \mathbb{R}$,

(ii) $S(t, \sigma) S(\sigma, \tau)=S(t, \tau)$, for any $t \geqslant \sigma \geqslant \tau$,

(iii) $(t, \tau) \mapsto S(t, \tau) v$ is continuous for all $t \geqslant \tau$ and $v \in \mathcal{X}$.

\section{Limiting consideration}

For each $t \in \mathbb{R}$ we consider the sesquilinear form

$$
a_{t}^{0}: H^{1}(\omega) \times H^{1}(\omega) \rightarrow \mathbb{R}
$$

defined by

$$
a_{t}^{0}(u, v)=\int_{\omega} g(x, t)(\nabla u \cdot \nabla v+u v) d x .
$$

With this definition we immediate have that

$$
\alpha_{1}\|v\|_{H^{1}(\omega)}^{2} \leq a_{t}^{0}(v, v) \leq \alpha_{2}\|v\|_{H^{1}(\omega)}^{2},
$$

for all $v \in H^{1}(\omega)$.

Similarly, $a_{t}^{0}$ gives rise a densely defined positive linear operator with compact resolvent,
$A_{0}(t): D\left(A_{0}(t)\right) \subset L^{2}(\omega) \rightarrow L^{2}(\omega)$, defined by relation

$a_{t}^{0}(u, v)=\left(\left(A_{0}(t) u, v\right)\right)_{t}, u \in H^{1}(\omega), v \in D\left(A_{0}(t)\right)$

where $((u, v))_{t}:=\int_{\omega} g(x, t) u v d x d y, u, v \in L^{2}(\omega)$.

By regularity of $\partial \omega$,

$$
D\left(A_{0}(t)\right)=\left\{v \in H^{2}(\omega): \nabla v \cdot \eta=0\right\},
$$

and is independent on $t$. Moreover

$A_{0}(t) v=-\frac{1}{g(\cdot, t)} \sum_{i=1}^{n}\left(g(\cdot, t) v_{x_{i}}\right)_{x_{i}}+v, v \in D\left(A_{0}(t)\right)$.

By $\left(G_{1}\right)-\left(G_{2}\right)$ there exists a constant $k_{2}$ (independent on $t$ ) such that

$\left|a_{t}^{0}(u, v)-a_{t}^{0}(u, v)\right| \leq k_{2}|t-s|\|u\|_{H^{1}(\omega)}\|v\|_{H^{1}(\omega)}$,

for all $t, s \in \mathbb{R}$ and $u, v \in H^{1}(\omega)$.

Therefore, writing equation (6) as an abstract evolution equation

$$
\left\{\begin{array}{l}
\frac{d v^{0}}{d t}(t)+A_{0}(t) v^{0}(t)=f^{e}\left(v^{0}(t)\right) \\
v^{0}(\tau)=v_{\tau}^{0} \in H^{1}(\omega)
\end{array}\right.
$$

we can define a (nonlinear) evolution process $\left\{S_{0}(t, \tau): t \geq \tau\right\}$ in the state space $H^{1}(\omega)$ by $S_{0}(t, \tau) v^{0}:=\overline{v^{0}}\left(t, \tau, v^{0}\right)$

Now we have now the elements to state our results

Lemma 2 If $\left\{f^{\epsilon}\right\}$ is a bounded family in $L^{2}(\Omega)$ then $\left\{A_{\epsilon}(t)^{-1} f^{\epsilon}\right\}$ is a bounded family in $H_{\epsilon}$.

Lemma 3 Let $\left\{f^{\epsilon}\right\}$ be a bounded family in $L^{2}(\Omega)$. If $M f^{\epsilon} \rightarrow \hat{f} w-L^{2}(\omega)$, then

$$
\left\|A_{\epsilon}(t)^{-1} f^{\epsilon}-E A_{0}(t)^{-1} \hat{f}\right\|_{H_{\epsilon}} \stackrel{\epsilon \rightarrow 0}{\longrightarrow} 0,
$$

uniformly in $t$ in bounded subsets of $\mathbb{R}$, where the extension operator $E$ is defined as

$$
\begin{gathered}
E: H^{1}(\omega) \rightarrow H^{1}(\Omega) \\
(E u)(x, y)=u(x)
\end{gathered}
$$

Theorem 4 Under the assumptions $(G 1),(G 2)$ on the profile $g \in C^{2}(\bar{\omega} \times \mathbb{R} ; \mathbb{R})$, and assuming that the nonlinearity $f \in C^{2}(\mathbb{R} ; \mathbb{R})$ is bounded with bounded derivatives up second order, then equations (12) and (14) generate evolution processes $\left\{S_{\epsilon}(t, \tau): t \geq \tau\right\}$ and $\left\{S_{0}(t, \tau): t \geq \tau\right\}$ in $H_{\epsilon}$ and $H^{1}(\omega)$ respectively. Moreover, given $v^{\epsilon} \in H_{\epsilon}$ and $v^{0} \in H^{1}(\omega)$ such that $v^{\epsilon} \stackrel{\epsilon \rightarrow 0}{\longrightarrow} E v^{0}$ in $L^{2}(\Omega)$, then

$$
\left\|S_{\epsilon}(t, \tau) v^{\epsilon}-E S_{0}(t, \tau) v^{0}\right\|_{H_{\epsilon}} \stackrel{\epsilon \rightarrow 0}{\longrightarrow} 0,
$$

uniformly for $(t, \tau), t \geq \tau$, in bounded subsets of $\mathbb{R}^{2}$.

Proof: See [5]. 


\section{Acknowledgment}

RPS is partially supported by FAPESP \#2012/06753-8 and FUNDUNESP \#0135812, Brazil.

\section{References}

[1] V.L. Carbone, M.J.D. Nascimento, K. Schiabel-Silva, R.P. Silva, Pullback attractors for a singularly nonautonomous plate equation, Electr. J. Differential Equations, 77, 113, (2011).

[2] J.K. Hale, Asymptotic Behavior of Dissipative Systems, Mathematical Surveys and Monographs, 25, AMS, (1988).

[3] P.E. Kloeden, P. Marín-Rubio, J. Real, Pullback attractors for a semilinear heat equation in a non-cylindrical domain, J. Differential Equations, 244, 2062-2091, (2008). DOI: 10.1016/j.jde.2007.10.031

[4] P.E. Kloeden, J. Real, C. Sun, Pullback attractors for a semilinear heat equation on time-varying domains, J. Differential Equations, 246, 4702-4730, (2009). DOI: 10.1016/j.jde.2008.11.017

[5] J.V. Pereira, R.P. Silva, Reactiondiffusion equations in a noncylindrical thin domain, submitted, (2013). http://www.rc.unesp.br/igce/matematica/ rpsilva/pre-prints/pre-prints/Pre-print.html

[6] H. Tanabe, Equations of Evolution, Pitman Publishing Limited, (1979). 